\title{
Green Synthesis of High Temperature Stable Anatase Titanium Dioxide Nanoparticles Using Gum Kondagogu: Characterization and Solar Driven Photocatalytic Degradation of Organic Dye
}

\author{
Kothaplamoottil Sivan Saranya ${ }^{1}$, Vinod Vellora Thekkae Padil ${ }^{2, *} \mathbb{1}$, Chandra Senan ${ }^{3}$, \\ Rajendra Pilankatta ${ }^{4}$, Kunjumon Saranya ${ }^{1}$, Bini George ${ }^{1, *}$, Stanisław Wacławek ${ }^{2}{ }^{\circledR}$ and \\ Miroslav Černík ${ }^{2, *}$ \\ 1 Department of Chemistry, School of Physical Sciences, Central University of Kerala, Kerala 671316, India; \\ sharanyacks@gmail.com (S.K.S.-K.S.S.); kunjumonsaranya916@gmail.com (S.K.-K.S.) \\ 2 Institute for Nanomaterials, Advanced Technologies and Innovation (CXI), Technical University of \\ Liberec (TUL), Studentská 1402/2, 46117 Liberec 1, Czech Republic; stanislaw.waclawek@tul.cz \\ 3 Centre for Water Soluble Polymers, Applied Science, Faculty of Arts, Science and Technology, \\ Wrexham Glyndwr University, Wrexham LL11 2AW, Wales, UK; c.senan@glyndwr.ac.uk \\ 4 Department of Biochemistry and Molecular Biology, School of Biological Sciences, Central, \\ University of Kerala, Kerala 671316, India; praj74@gmail.com \\ * Correspondence: vinod.padil@tul.cz (V.V.T.P.); binigeorgek@gmail.com (B.G.); miroslav.cernik@tul.cz (M.Č.); \\ Tel.: +420-723-372-911 (V.V.T.P. \& M.Č.)
}

Received: 28 October 2018; Accepted: 1 December 2018; Published: 4 December 2018

\begin{abstract}
The present study reports a green and sustainable method for the synthesis of titanium dioxide $\left(\mathrm{TiO}_{2}\right)$ nanoparticles (NPs) from titanium oxysulfate solution using Kondagogu gum (Cochlospermum gossypium), a carbohydrate polymer, as the NPs formation agent. The synthesized $\mathrm{TiO}_{2} \mathrm{NPs}$ were categorized by techniques such as X-Ray Diffraction (XRD), Fourier transform infrared (FTIR) spectroscopy analysis, Raman spectroscopy, scanning electron microscope- Energy-dispersive X-ray spectroscopy (SEM-EDX), Transmission electron microscopy (TEM), High-resolution transmission electron microscopy (HR-TEM), UV-visible spectroscopy, Brunauer-Emmett-Teller (BET) surface area and particle size analysis. Additionally, the photocatalytic actions of $\mathrm{TiO}_{2} \mathrm{NPs}$ were assessed with regard to their ability to degrade an organic dye (methylene blue) from aqueous solution in the presence of solar light. Various parameters affecting the photocatalytic activity of the $\mathrm{TiO}_{2} \mathrm{NPs}$ were examined, including catalyst loading, reaction time, $\mathrm{pH}$ value and calcination temperature of the aforementioned particles. This green synthesis method involving $\mathrm{TiO}_{2} \mathrm{NPs}$ explores the advantages of inexpensive and non-toxic precursors, the $\mathrm{TiO}_{2} \mathrm{NPs}$ themselves exhibiting excellent photocatalytic activity against dye molecules.
\end{abstract}

Keywords: titanium dioxide nanoparticles; green synthesis; gum kondagogu; methylene blue; photocatalysis

\section{Introduction}

Nanoparticles (metal and metal oxides) of various types have been widely employed via physical and chemical methods. Although these systems have resulted in the formation of numerous extremely diverse nanostructures, many environmental toxicity issues have emerged [1]. Metal oxides have recently been widely explored because of the huge variety of structural, material and functional properties exhibited by their nanoparticles. Transition metal oxide nanoparticles have generated 
great scientific interest owing to their unusual properties compared with their corresponding bulk metals. Moreover, they have numerous industrial applications [2]. Metal oxide nanoparticles with disparate morphologies and sizes have been synthesized using different synthetic routes. These include hydrothermal, solvothermal, microwave, vapour deposition, spray pyrolysis and wet-chemical methods [3-5]. However, the usage of solvents in the chemical synthesis route adopted pose limitations for the application of NPs in medicine, pharmacy and other areas, primarily due to the toxicity caused by the solvents. Thus, there is an urgent need for the development of alternative, novel nanoparticle synthesis processes that could be exploited (at both the industrial and commercial level) in order to introduce cleaner, safer and smarter products suitable for application in communication technologies, medicine, pharmacy, agriculture and other industries. The introduction of environmentally benign approaches for designing NPs provides solutions to mounting tasks related to ecological concerns. Use of greener, safe and environmentally non-hazardous chemicals and green protocols for the synthesis of nanoparticles decreases the expense of synthesis while minimizing the utilization of harmful substances and their subsequent disposal [6].

Titanium dioxide $\left(\mathrm{TiO}_{2}\right)$ is regarded as an extremely promising metal oxide that can perform a key role in solving the global energy crunch, as well as serving to assuage environmental concerns $[7,8]$. Nanoparticles of $\mathrm{TiO}_{2}$ have featured in numerous photovoltaic applications and in various sectors including cosmetic, pharmaceutical and skin care products. These versatile NPs can also confer whiteness to paints, plastics, papers, inks, food colorants and toothpaste while also finding usage in cancer treatment $[9,10]$. The synthesis of $\mathrm{TiO}_{2} \mathrm{NPs}$, which possess controlled crystal phases and morphologies that make them eminently suitable in diverse applications requiring high performance, has proven to be fundamentally challenging for the scientific community [11].

Carbohydrate polymers isolated from trees [12], are an innovative type of potentially cost-effective and biologically favorable biomaterials that display highly particular and selective characteristics towards the design of nanostructures for unique applications. They are natural biopolymers based on plant exudates and possess interesting properties e.g., reducing, stabilizing, suspending, gelling etc. [13-15]. Tree exudates (gums) serve as 'green' media and harbor numerous hydroxyl, carbonyl and carboxylic functional groups. Such functional groups can act as good chemical reductants and their presence in these gum hydrocolloid materials facilitates the formation of metal or metal oxide NPs. This is achieved in two ways: either through the reduction of metal ions or by the gum molecules behaving as a stabilizing mediator (to prevent nanoparticle agglomeration). Hence, the essential criterion for designing nanomaterials with desirable properties is fulfilled [16,17].

The Kondagogu (Cochlospermum gossypium, KG) is a native tree growing in the forests of India and its exudates have been categorized as being substituted rhamnogalacturonans [16]. KG is a complex and acidic polysaccharide with high solution viscosity and gelation characteristics. KG contains sugars such as arabinose, rhamnose, glucose, galactose, mannose, glucuronic acid and galacturonic acid. The structural features assigned to KG are $(1 \rightarrow 2) \beta$-D-Gal $p,(1 \rightarrow 6) \beta$-D-Gal $p,(1 \rightarrow 4) \beta$-D-Glcp A, 4-O-Me- $\alpha$-D-Glcp A and $(1 \rightarrow 2) \alpha$-L-Rha $[18,19]$. It has a high content of uronic acid and diverse functional groups (hydroxyl, acetyl, carbonyl and carboxylic) $[18,20]$. The morphological, physicochemical and structural analysis of this biopolymer is currently being extensively researched $[18,19]$.

Organic dyes represent major clusters of pollutants flushed out into wastewaters from textiles and other industrialized procedures. Due to their potential injuriousness and presence in external waters, their elimination and removal has been a matter of considerable importance, since even small amounts of released dyes can discolor surface waters and impact negatively on the otherwise aesthetic and pristine surroundings [21]. The dyes altered the absorption and reflection of sunlight entering the water, which in turn affected bacterial growth and subsequently the level of biological impurities in the water [22]. Wastewater purification is discernible as one of the most serious environmental challenges of the present day. To this end, solid catalysts have been widely used in various water treatment technologies, both in processing and industry. Nanoparticles, courtesy of their small size and high 
surface-to-volume ratios, display high absorbing, interacting and reactive capabilities, underscoring their value in wastewater remediation [23].

Photocatalysis is an environmentally benign process involving the conversion of light energy into chemical energy under ambient conditions. The outstanding performance of solar driven photocatalytic processes in solving environmental problems has gained much attention in recent years, effluents from the textile and paint industries being the main sources of environmental organic contaminants. Thus, it is essential that the latter is degraded and converted into harmless mineral compounds. The photocatalyst can be harnessed for environmental remediation, which includes abstraction of pesticides, fungicides and fertilizers from wastewater and the eradication of organic pollutants from the air [24]. Activation of a semiconductor photocatalyst is achieved by the absorption of a photon, which results in the transfer of an electron from the valence band to the conduction band by creation of a hole $\left(\mathrm{h}^{+}\right)$in the valence band [25]. These photo-generated charge carriers cause redox reactions on the surface of the photocatalyst, i.e., any contaminant that is adsorbed onto the photocatalyst surface will undergo reduction or oxidation by the electron-hole pair respectively. Generally, the metal oxide photocatalyst surface acts as an active center in photocatalysis, either by the generation of $\mathrm{OH}$. radicals (by oxidation of $\mathrm{OH}^{-}$anions) or by the generation of $\mathrm{O}_{2}{ }^{-}$radicals (by the reduction of $\mathrm{O}_{2}$.) Subsequently, these photo-generated radicals and anions react with the adsorbed organic contaminants, degrading or mineralizing them into less harmful by-products. The photocatalytic reaction can be employed to bring about the transformation of highly toxic chemicals into less noxious or non-toxic products such as $\mathrm{CO}_{2}$ and $\mathrm{H}_{2} \mathrm{O}[26,27]$. Consequently, metal oxides can be employed as prime candidates for the effective photocatalytic degradation of environmental contaminants.

In this study, we have focused on the green synthesis of $\mathrm{TiO}_{2} \mathrm{NPs}$ and their effective application in the photocatalytic degradation of a commercially used organic dye, methylene blue (MB). The influence of various solution $\mathrm{pH}$ values on photocatalytic efficiency was also studied.

\section{Materials and Methods}

\subsection{Materials}

Titanium oxysulfate and methylene blue were purchased from Sigma Aldrich and HiMedia Chemicals, Mumbai, India, respectively. KG samples were obtained from the Girijan Co-operative Corporation Ltd. (GCC), Hyderabad, India. All the other chemicals and solvents used were of analytical grade.

\subsection{Fabrication of $\mathrm{TiO}_{2}$ Nanoparticles}

Titanium dioxide NPs were synthesized using a typical procedure as described here. The procedure involved adding KG $(50 \mathrm{mg})$ to $10 \mathrm{~mL}$ of titanium oxysulfate $(0.1 \mathrm{M})$ and stirring vigorously $(750 \mathrm{rpm})$ on a magnetic stirrer at $90-95^{\circ} \mathrm{C}$. Later, the product was centrifuged, washed and dried. The dried sample was calcined at $500{ }^{\circ} \mathrm{C}$ for $4 \mathrm{~h}$, then pulverized and stored until further use.

\subsection{Characterization of $\mathrm{TiO}_{2}$ Nanoparticles}

\subsubsection{X-Ray Diffraction (XRD) Analysis}

$X$-Ray Diffraction (XRD) configurations of the calcined samples were obtained with the diffraction

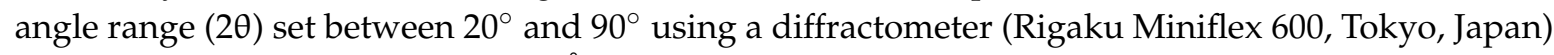
with nickel filtered $\mathrm{Cu} \mathrm{K} \alpha(\lambda=1.54 \AA)$ radiation and a liquid nitrogen cooled, germanium solid state detector. The spectral plots were compared with details obtained from Joint Committee on Powder Diffraction Standards (JCPDS), data files for analytical purposes. 


\subsubsection{FTIR Analysis}

For the Fourier transform infrared spectroscopy (FTIR) analysis, a spectrometer (Perkin-Elmer FTIR Spectrum Two, Singapore) in attenuated total reflection (ATR) mode and with the spectral range set between 4000 and $400 \mathrm{~cm}^{-1}$ and a resolution of $4 \mathrm{~cm}^{-1}$ was used.

\subsubsection{Raman Spectra}

A Raman microscope (NICOLET DXR, Thermo Scientific, Waltham, MA, USA), equipped with an optical microscope, was used. An argon-ion $(532 \mathrm{~nm})$ or helium-neon $(632.8 \mathrm{~nm})$ laser was used for the excitation of the Raman signal with appropriate holographic notch filters to eliminate the laser line after excitation. Spectral analysis and curve fitting were performed using GRAMS/AI 8.00 Spectroscopy software (Alfasoft GmbH, Frankfurt, Germany)

\subsubsection{Scanning electron microscope- Energy-dispersive X-ray spectroscopy (SEM-EDX) Analysis}

The elemental composition and morphology of $\mathrm{TiO}_{2}$ nanoparticles were determined using a scanning electron microscope (ZEISS, Ultra/Plus, Potsdam, Germany). Energy-dispersive X-ray spectroscopy (EDX) measurements were carried out using a scanning electron microscope equipped with an EDX attachment (JSM-6390 172, JEOL, Tokyo, Japan).

\subsubsection{TEM and High-resolution transmission electron microscopy (HR-TEM) Analysis}

The nanoparticles and particle distributions determined were recorded by a transmission electron microscopy (TEM) (JEOL, JEM-2100,Tokyo, Japan). The samples were prepared on standard copper TEM grids covered with thin carbon foil. Drops of $\mathrm{TiO}_{2}$ nanoparticles were dispersed in $1 \mathrm{~mL}$ of isopropanol using ultrasound for $10 \mathrm{~min}$. and a drop of the resulting solution was gently spread onto the upper surface of the carbon covered copper TEM grid.

\subsubsection{Particle Size Analysis}

Nanoparticle size distributions were measured by centrifugal particle sedimentation (CPS) using the Disc Centrifuge technique (DC24000UHR, CPS Instruments Inc., Prairieville, LA, USA).

\subsubsection{BET Surface Area}

The surface area of the $\mathrm{TiO}_{2}$ NPs was analyzed using the Brunauer-Emmett-Teller (BET) technique (Autosorb iQ, Quantachrome Instruments, Boynton Beach, FL, USA).

\subsubsection{Thermal Stability}

Thermal properties of $\mathrm{KG}$ and green-synthesized $\mathrm{TiO}_{2}$ nanoparticles were studied using thermogravimetric analysis (TGA) by means of a Perkin Elmer STA 6000 Thermal Analyzer (Singapore) instrument.

\subsubsection{Optical Properties}

Optical properties were determined using an ultraviolet (UV)-visible spectrophotometer (Perkin Elmer Lambda 35, Singapore) over the spectral region 200-800 nm.

\subsection{Photocatalytic Degradation of Methylene Blue}

The photocatalytic activity of green-synthesized $\mathrm{TiO}_{2}$ nanoparticles was scrutinized by the degradation of MB under sunlight. Photocatalytic activity in the presence of sunlight was determined under direct normal sunlight at an intensity of 100,000 Lux and the solar intensity measurement was carried out throughout the experiment at different time intervals. The degradation of dye was examined by collecting $5 \mathrm{~mL}$ aliquots from the reaction mixture at different intervals of time. 
In addition, these aliquots were centrifuged at $7000 \mathrm{rpm}$ for $15 \mathrm{~min}$. Photocatalytic degradation of the dye was monitored by measuring the absorbance spectra of the supernatants using a UV-visible spectrophotometer over the wavelength range $200-800 \mathrm{~nm}$.

\subsubsection{Photocatalytic Studies Based on Catalyst Concentration}

In order to study the effect of catalyst loading on solar light driven photocatalysis of methylene blue, different amounts (1-15 mg) of green-synthesized titanium dioxide nanoparticles calcined at $500{ }^{\circ} \mathrm{C}$ were added to $50 \mathrm{~mL}$ of dye solution. The reaction suspension was mixed thoroughly using a magnetic stirrer for $90 \mathrm{~min}$ in the presence of sunlight. The photocatalytic degradation of the dye was monitored by measuring the absorbance of the solution at regular intervals using a UV-visible spectrophotometer.

\subsubsection{Photocatalytic Studies Based on Time}

In a typical experiment, $50 \mathrm{~mL}$ of dye solution $\left(1.0 \times 10^{-5} \mathrm{M}\right)$ was mixed with $10 \mathrm{mg}$ of $\mathrm{TiO}_{2}$ nanoparticles. The mixture was stirred continuously at $600 \mathrm{rpm}$ in the presence of sunlight. The rate of degradation was monitored by measuring the absorbance of the solution (over the 200 to $800 \mathrm{~nm}$ wavelength range) with a UV-visible spectrophotometer by removing and monitoring $5 \mathrm{~mL}$ aliquots at defined time intervals. The process was continued for $90 \mathrm{~min}$.

\subsubsection{Photocatalytic Studies Based on $\mathrm{pH}$}

The influence of $\mathrm{pH}$ on solar driven photocatalytic activity of the catalyst was studied by conducting the experiment at different $\mathrm{pH}$ values (4-9) of dye solution. Green-synthesized titanium dioxide nanoparticles $(10 \mathrm{mg})$ calcined at $500{ }^{\circ} \mathrm{C}$ were added to $50 \mathrm{~mL}$ of the dye solution having different $\mathrm{pH}$ values. The resulting suspension was thoroughly mixed using a magnetic stirrer for $90 \mathrm{~min}$ in the presence of sunlight and the absorbance of the solution measured with a UV-visible spectrophotometer.

\subsubsection{Photocatalytic Studies Based on Temperature}

The green-synthesized $\mathrm{TiO}_{2}$ nanoparticles were calcined at different temperatures $\left(500-900{ }^{\circ} \mathrm{C}\right.$ ) and each sample $(10 \mathrm{mg})$ was added to $50 \mathrm{~mL}$ of dye solution. The resulting suspension was thoroughly mixed using a magnetic stirrer for $90 \mathrm{~min}$, in the presence of sunlight. The absorbance of the solution was measured with a UV-visible spectrophotometer.

\section{Results and Discussion}

\subsection{Mechanism of $\mathrm{TiO}_{2}$ NPs Formation Via Green Synthesis}

Natural tree based carbohydrate polymers-an environmentally benign medium-contain extensive numbers of hydroxyl, carbonyl and carboxylic groups which can act as good chemical reductants. The presence of these functional groups in the gum hydrocolloid material facilitates the formation of metal nanoparticles. When a metal oxide precursor is introduced into a well dissolved KG homogeneous solution or hydrogel, the polyhydroxylated macromolecules inherent in the gum matrix are able to absorb metal cations. For example, the $\mathrm{Ti}^{3+}$ ion could be chelated with $\mathrm{KG}$ by means of $-\mathrm{OH}$ and $-\mathrm{COOH}$ groups. The sequestration of cations $\left[\mathrm{M}^{n+}\right]$ or hydroxylated cations $[\mathrm{M}(\mathrm{OH})]^{m+}$ that can undergo nucleation or growth processes is accelerated by the highly reactive hydroxyl groups present in KG. The last step in the $\mathrm{TiO}_{2}$ nanoparticle synthesis is the calcination process at $500{ }^{\circ} \mathrm{C}$ which removes the gum template via combustion of their organic scaffold, giving rise to the formation of dispersed oxides $[11,28]$. While XRD and Raman spectroscopy confirmed the formation of anatase as the only distinguished mineral phase; FTIR, TGA and EDX collectively proved that no organic compounds (polysaccharides, hydroxyl, carboxylate and other oxidic groups) were present in the final nanoparticles. 


\subsection{XRD Analysis}

The XRD pattern of $\mathrm{TiO}_{2} \mathrm{NPs}$ calcined at $500{ }^{\circ} \mathrm{C}$ showed a dominant peak at the $2 \theta$ value of $25.25^{\circ}$ (Figure 1). This matches the (101) crystallographic plane of the $\mathrm{TiO}_{2}$ anatase structure, indicating that the crystal composition is predominantly anatase. The other characteristic diffraction peaks were at the following $2 \theta$ values: $37.75,48.02,53.86,54.95,62.63,68.88,70.28,75.13$ and $82.60^{\circ}$. These values correspond to (004), (200), (105), (211), (204), (116), (220), (215) and (224) crystallographic planes of anatase, respectively (JCPDS No. 01-071-1166), thus confirming the formation of $\mathrm{TiO}_{2}$ nanoparticles in the anatase phase [29]. The average crystallite size of the NPs [i.e., the mean size of the ordered (crystalline) domains, which may be smaller or equal to the grain size] was calculated using the Scherrer formula $[d=0.89 \lambda / \beta \cos \theta]$ to be $12.58 \mathrm{~nm}$. The crystallinity and higher purity of prepared $\mathrm{TiO}_{2}$ NPs in the anatase form was validated by the presence of sharp peaks while the absence of peaks represented other crystallite forms of $\mathrm{TiO}_{2}$ [30-32].

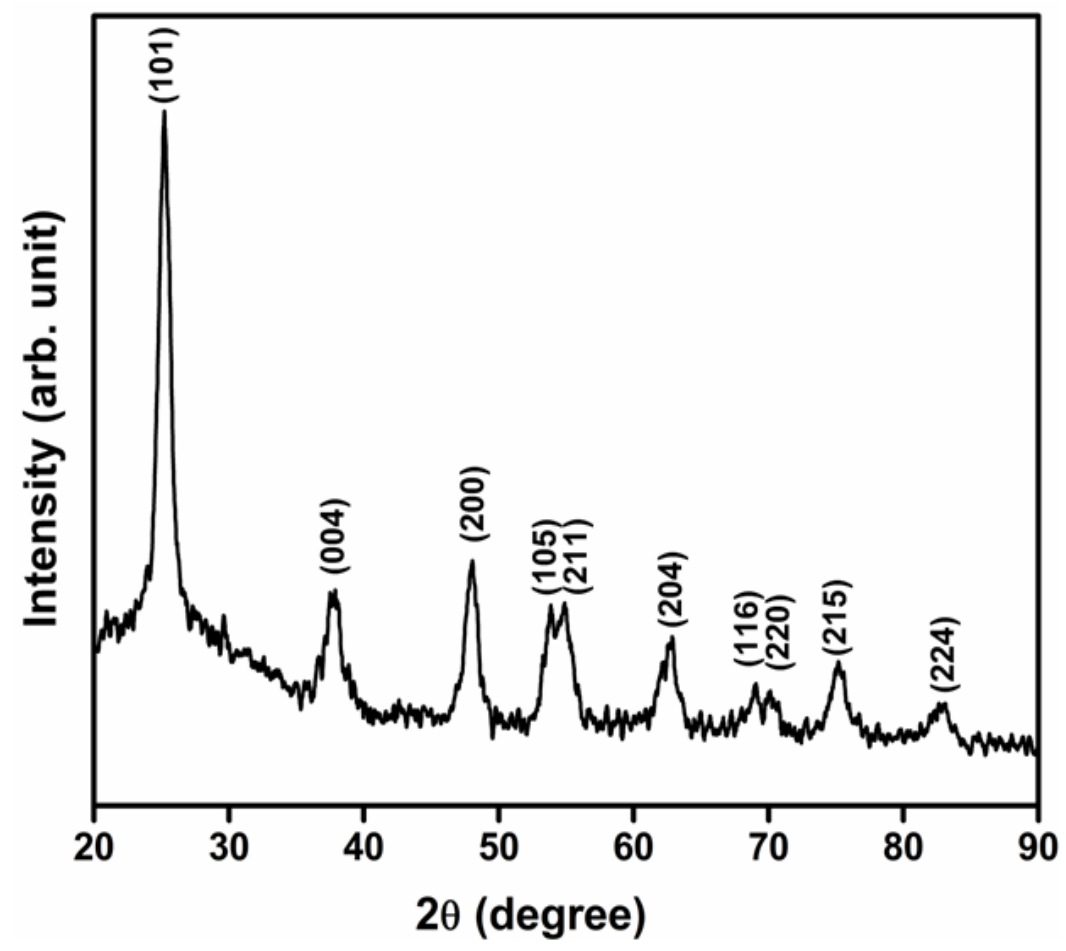

Figure 1. X-ray diffraction analysis (XRD) pattern of titanium dioxide $\left(\mathrm{TiO}_{2}\right)$ nanoparticles calcined at $500^{\circ} \mathrm{C}$.

XRD patterns of $\mathrm{TiO}_{2}$ nanocatalysts calcined at various temperatures are shown in Figure 2. When the calcination temperatures did not exceed $700{ }^{\circ} \mathrm{C}$, anatase was the only phase, with Joint Committee on Powder Diffraction Standards (JCPDS) No. 01-071-1166, while phase transformation of $\mathrm{TiO}_{2}$ was observed at $800{ }^{\circ} \mathrm{C}$. The rutile phase appeared in the $800^{\circ} \mathrm{C}$ sample and became the dominant phase in the $900{ }^{\circ} \mathrm{C}$ sample with peaks at $2 \theta=27.4^{\circ}(110), 36.04^{\circ}(101), 41.2^{\circ}(111), 54.3^{\circ}(211)($ JCPDS no. 01-073-2224). Formation of the rutile phase of $\mathrm{TiO}_{2}$ is normally observed above $600{ }^{\circ} \mathrm{C}$ with a complete transformation to the rutile form occurring at $800{ }^{\circ} \mathrm{C}$ [33]. The present XRD patterns illustrate that the anatase to rutile phase transformation of the synthesized $\mathrm{TiO}_{2}$ first took place at $800{ }^{\circ} \mathrm{C}$ and was almost completed at $900{ }^{\circ} \mathrm{C}$, thus revealing the formation of a high temperature, stable anatase phase of $\mathrm{TiO}_{2}$ nanocatalysts through the green synthesis method adopted. 


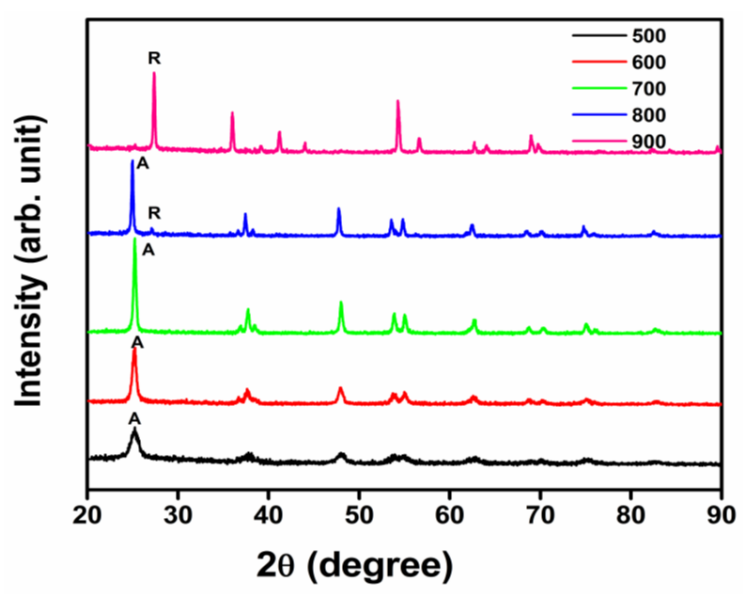

Figure 2. XRD pattern of $\mathrm{TiO}_{2}$ nanoparticles calcined at different temperatures ( $\mathrm{R}$ denotes rutile and $\mathrm{A}$ denotes anatase phases of $\mathrm{TiO}_{2}$ nanoparticles).

While anatase and rutile are both mineral forms of titanium dioxide (possessing tetragonal crystal systems), in terms of optical activity, anatase is optically negative whereas rutile is positive. Furthermore, the luster exhibited by anatase is more strongly adamantine or metallic-adamantine than that shown by rutile.

\subsection{Fourier Transform Infrared Spectroscopy Analysis}

FTIR spectroscopy was employed to identify different functional groups present in KG and on the surface of the formed NPs (Figure 3). The major stretching frequencies in the spectrum for KG were observed at 3368,1719,1609,1417,1247, 1145 and $1035 \mathrm{~cm}^{-1}$. The band observed at $3368 \mathrm{~cm}^{-1}$ suggests the presence of hydroxyl groups while those noted at $1719 \mathrm{~cm}^{-1}$ and $1609 \mathrm{~cm}^{-1}$ were ascribed to carbonyl stretching vibrations and the asymmetric stretching of carboxylate, respectively. The band seen at $1417 \mathrm{~cm}^{-1}$ was due to the symmetrical stretching of the carboxylate group present in the gum's uronic acid. The presence of an acetyl group was inferred by the band appearing at $1247 \mathrm{~cm}^{-1}$ while the bands registered at 1145 and $1035 \mathrm{~cm}^{-1}$ were indicative of $\mathrm{C}-\mathrm{O}$ stretching vibrations of ether and alcohol groups, respectively [34]. The absence of these characteristic peaks in the FTIR spectra of $\mathrm{TiO}_{2}$ nanoparticles promoted by KG may be a consequence of the higher purity of prepared nanoparticles in anatase crystal formations on calcination at $500{ }^{\circ} \mathrm{C}$. The characteristic signal for $\mathrm{TiO}_{2}$ nanoparticles observed below $1000 \mathrm{~cm}^{-1}$ in the FTIR spectra was due to Ti-O-Ti vibrations [35].

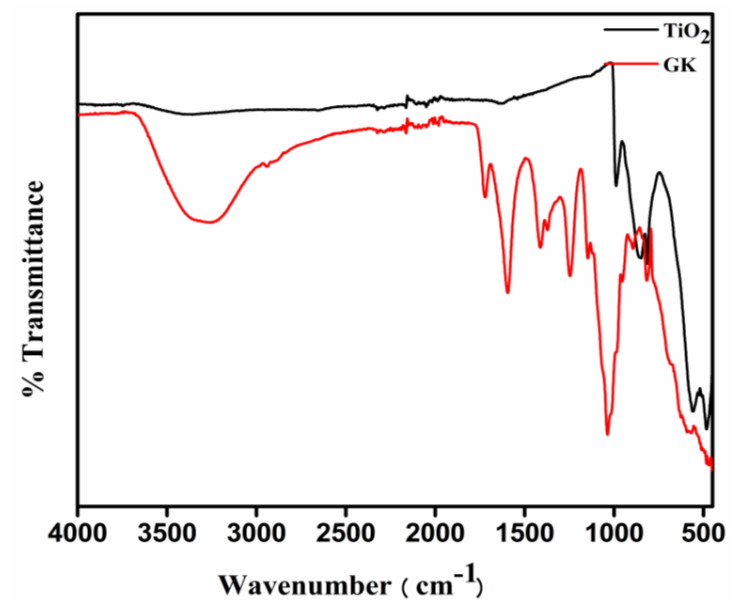

Figure 3. Fourier transform infrared spectroscopy (FTIR) spectra of Kondagogu (KG) and $\mathrm{TiO}_{2}$ nanoparticles. 


\subsection{Raman Spectroscopy}

To identify and quantify both the amorphous and crystalline $\mathrm{TiO}_{2}$ phases, Raman spectroscopy was employed. Figure 4 reveals that $\mathrm{TiO}_{2}$ anatase displays Raman bands at 639, 516, 399 and $197 \mathrm{~cm}^{-1}$, as well as a precise sharp and intense peak at $144 \mathrm{~cm}^{-1}$. According to the literature, the anatase phase of $\mathrm{TiO}_{2}$ has six Raman active modes, namely $A_{1 g}+2 B_{1 g}+3 E_{g}$, determined by group analysis $D_{4 h}$ (I4l/amd) [36], and known in the first-order Raman spectrum of single crystal $\mathrm{TiO}_{2}$ at $144 \mathrm{~cm}^{-1}$ $\left(E_{g}\right), 197 \mathrm{~cm}^{-1}\left(E_{g}\right), 399 \mathrm{~cm}^{-1}\left(B_{1 g}\right), 516 \mathrm{~cm}^{-1}\left(A_{1 g}+B_{1 g}\right)$ and $639 \mathrm{~cm}^{-1}\left(E_{g}\right)$ [37].

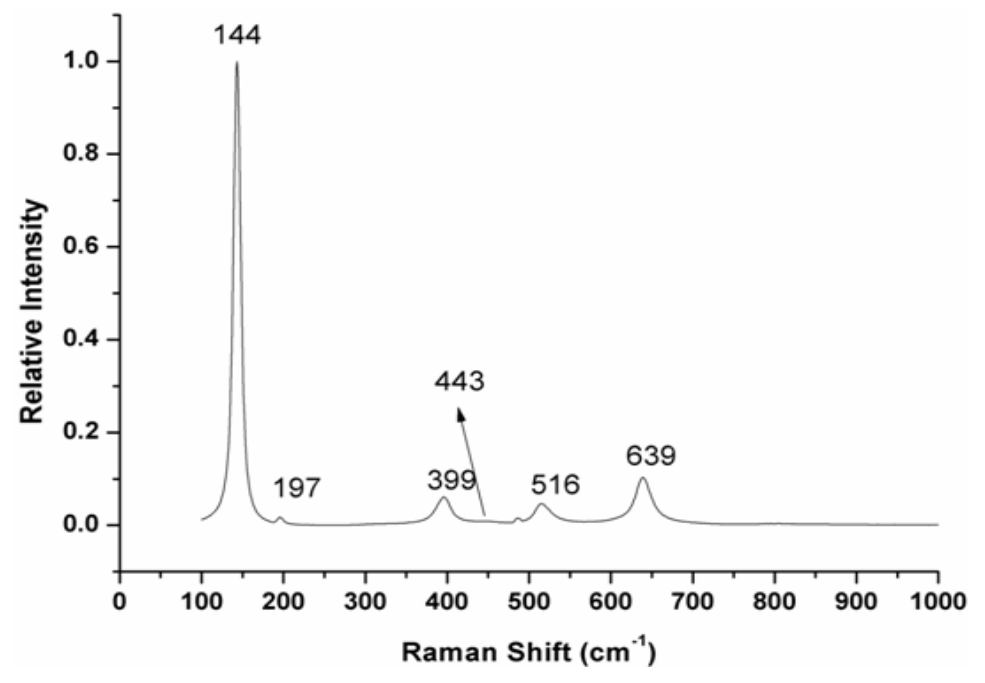

Figure 4. Raman spectrum of $\mathrm{TiO}_{2}$ nanoparticles.

\subsection{Scanning Electron Microscopy (SEM)}

The SEM micrograph of $\mathrm{TiO}_{2}$ nanoparticles is shown in Figure 5a, and the resulting nanoparticles were observed to have a spherical morphology. The chemical composition, analyzed using EDX spectra, confirmed the presence of Ti and $\mathrm{O}$ (Figure $5 \mathrm{~b}$ ). Since the sample was coated on a copper grid, peaks corresponding to $\mathrm{Cu}$ were also visible in Figure $5 \mathrm{~b}$.
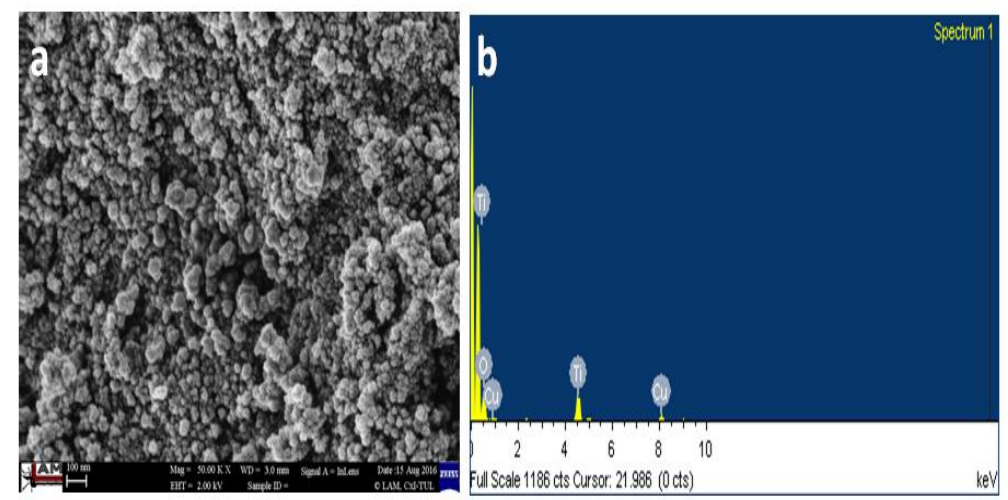

Figure 5. Typical (a) scanning electron microscopy (SEM) and (b) EDX (Energy Dispersive X-Ray) analysis of $\mathrm{TiO}_{2}$ nanoparticles.

\subsection{TEM and HR-TEM Analysis}

Both particle size and morphology of $\mathrm{TiO}_{2}$ were confirmed by TEM analysis (Figure 6a) which revealed that the particles are monodisperse and spherical in shape. The sizes of particles were in the 8-13 nm range and the selected area electron diffraction (SAED) pattern indicated that the $\mathrm{TiO}_{2}$ nanoparticles possessed good crystallinity (Figure 6b). HR-TEM observations (Figure 6c) suggest that 
the $\mathrm{TiO}_{2}$ nanoparticles have a perfect lattice structure. The particle sizes determined by the TEM image were similar to the reported values obtained by applying the Scherrer equation to the XRD patterns $(12.58 \mathrm{~nm})$. The aforementioned equation correlates the size of sub-micrometre particles or crystallites (in a solid) to the broadening of a peak in a diffraction pattern. It is employed for the determination of the size of particles of crystals in the form of powders.

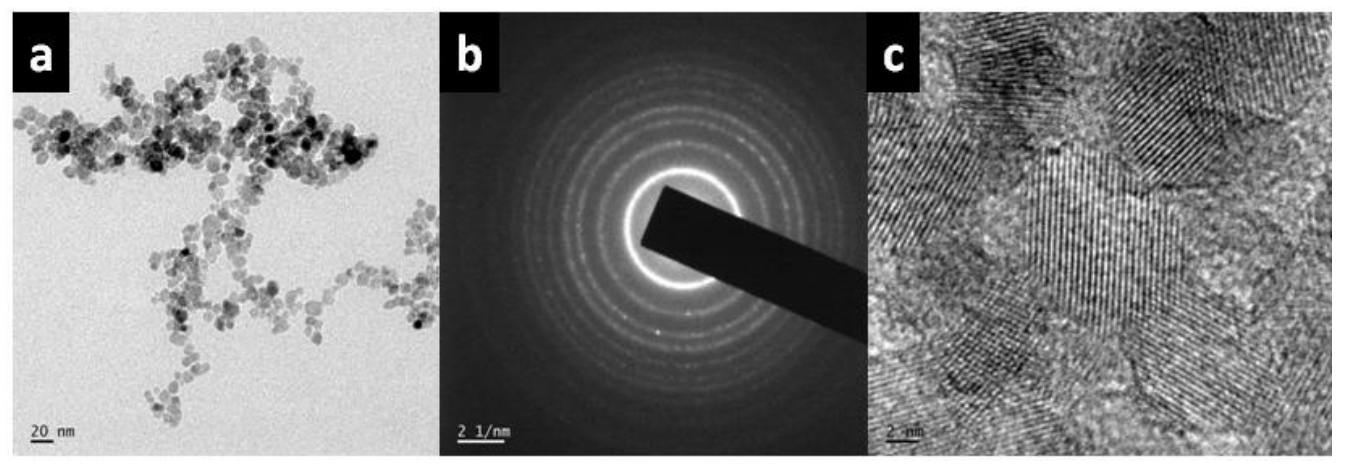

Figure 6. (a) TEM (Transmission Electron Microscopy) image, (b) SAED pattern and (c) HR-TEM (High Resolution TEM) micrograph of $\mathrm{TiO}_{2}$ nanoparticles.

\subsection{Particle Size E BET Analysis}

The $\mathrm{TiO}_{2}$ particle size distribution analyzed by CPS determined the mean size to be $11.2 \pm 0.2 \mathrm{~nm}$ (Figure 7). Evidently, the $\mathrm{TiO}_{2}$ nanoparticles appeared to be more stable in the current study involving green synthesis and the particle size measurements corresponded very well with the TEM analytical data (Figure 6). The specific surface area of $\mathrm{TiO}_{2}$ was determined from the isotherms to be $42.6 \mathrm{~m}^{2} / \mathrm{g}$ based on the BET analysis.

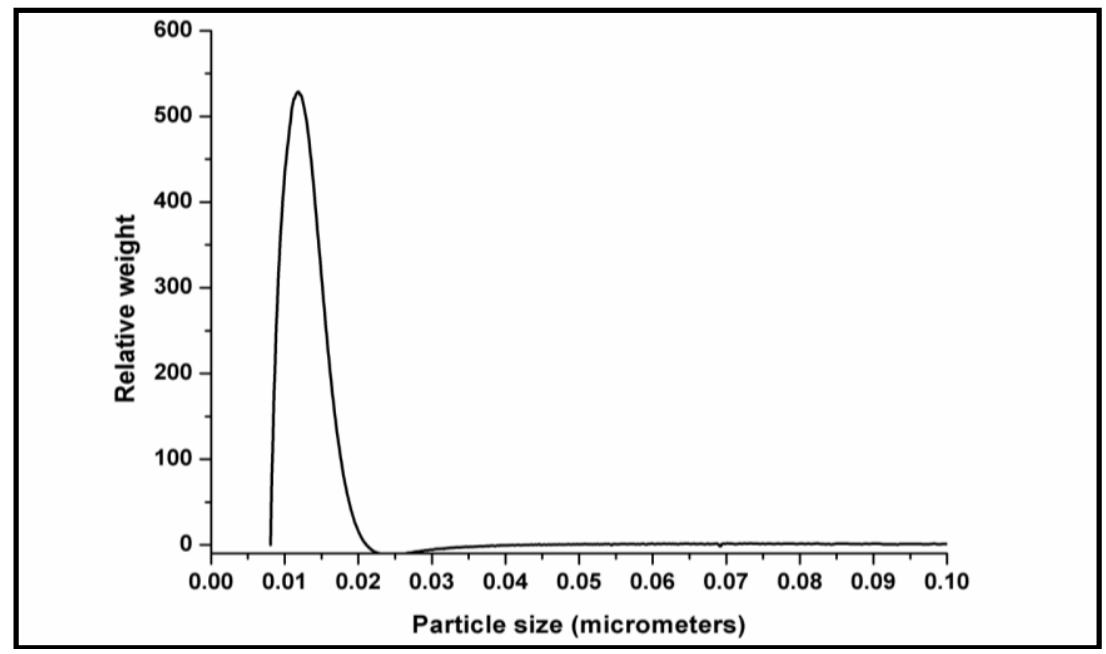

Figure 7. Particle size distributions of $\mathrm{TiO}_{2}$ nanoparticles as determined by the centrifugal particle sedimentation (CPS) method.

\subsection{Thermal Studies}

Thermal properties of $\mathrm{KG}$ and green-synthesized $\mathrm{TiO}_{2}$ nanoparticles were studied using thermogravimetric analysis. Depicted in Figure 8 are the TGA curves of $\mathrm{KG}$ and $\mathrm{TiO}_{2}$ nanoparticles heated from $35^{\circ} \mathrm{C}$ to $950{ }^{\circ} \mathrm{C}$. In the case of $\mathrm{KG}$, there were two major weight loss events. The first occurrence (observed between $35^{\circ} \mathrm{C}$ and $111^{\circ} \mathrm{C}$ ) of approximately $17 \%$ probably represented the loss of adsorbed water - as hydrogen bonded water - from the polysaccharide structure. The second weight loss event of roughly $33 \%$ was very significant and was discerned between 232 and $309{ }^{\circ} \mathrm{C}$. 
It was ascribed to decomposition of the polysaccharide. A third, far smaller weight loss instance was registered at $590{ }^{\circ} \mathrm{C}$, possibly due to the conversion of the remaining polymer to carbon residue [20].

The TGA curve of $\mathrm{TiO}_{2}$ (Figure $7 \mathrm{~b}$, red curve) did not indicate any weight loss up to $766^{\circ} \mathrm{C}$, a reflection of its high thermal stability, probably due to four hours of calcination at $500{ }^{\circ} \mathrm{C}$. There were small linear weight reductions detected in the range of $766-911^{\circ} \mathrm{C}$, possibly caused by the loss of residual carbon from the gum matrix.

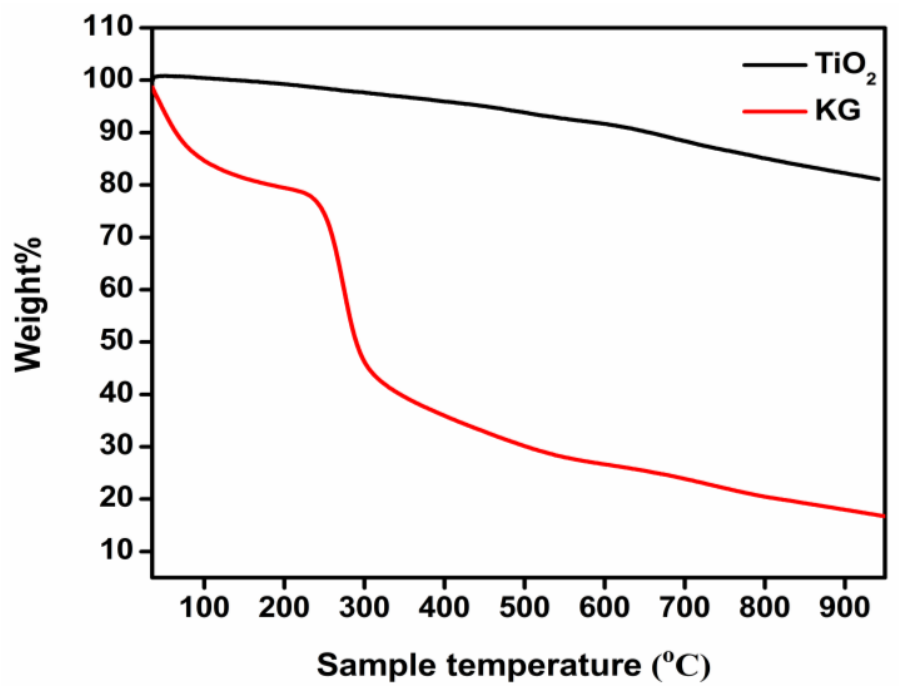

Figure 8. Thermogravimetric analysis (TGA) curves of $\mathrm{KG}$ and green-synthesized $\mathrm{TiO}_{2}$ nanoparticles.

\subsection{Optical Properties}

The UV-visible absorption spectrum of biosynthesized $\mathrm{TiO}_{2}$ nanoparticles is shown in Figure 8. The observed absorption spectrum matches those obtained with $\mathrm{TiO}_{2}$ nanoparticles produced by chemical methods, displaying a broad absorption band in the UV region, up to $380 \mathrm{~nm}$ [38].

The UV-visible spectrum was utilized to deduce the optical absorption properties of green-synthesized $\mathrm{TiO}_{2}$ nanoparticles (Figure 9). The band gap energy of green-synthesized $\mathrm{TiO}_{2}$ nanomaterials was found to be $3.13 \mathrm{eV}$ from the Tauc plot used to determine the optical bandgap in semiconductors as shown in Figure 10.

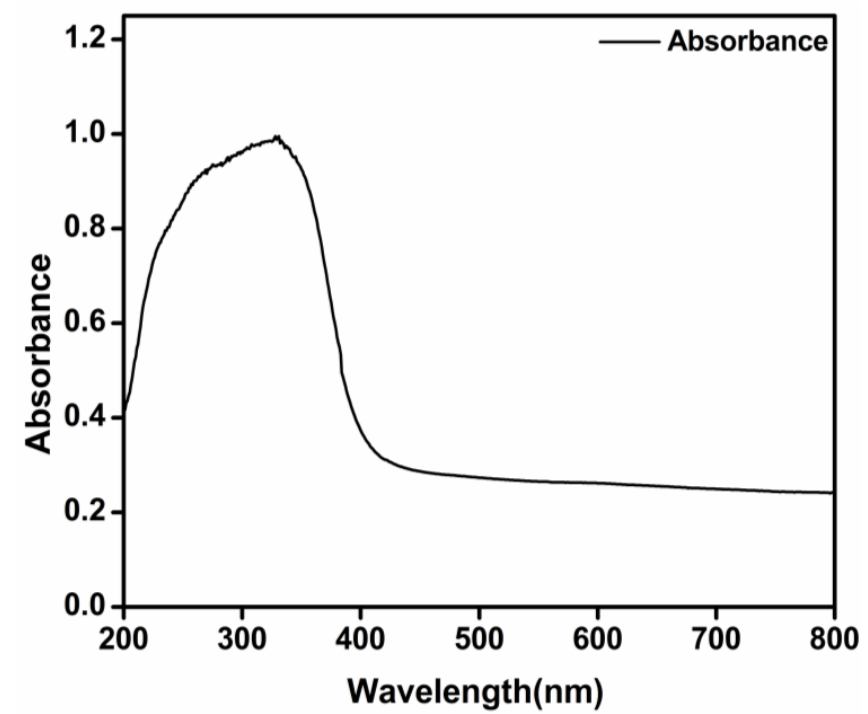

Figure 9. Ultraviolet (UV)-visible absorption spectrum of green-synthesized $\mathrm{TiO}_{2}$ nanoparticles . 


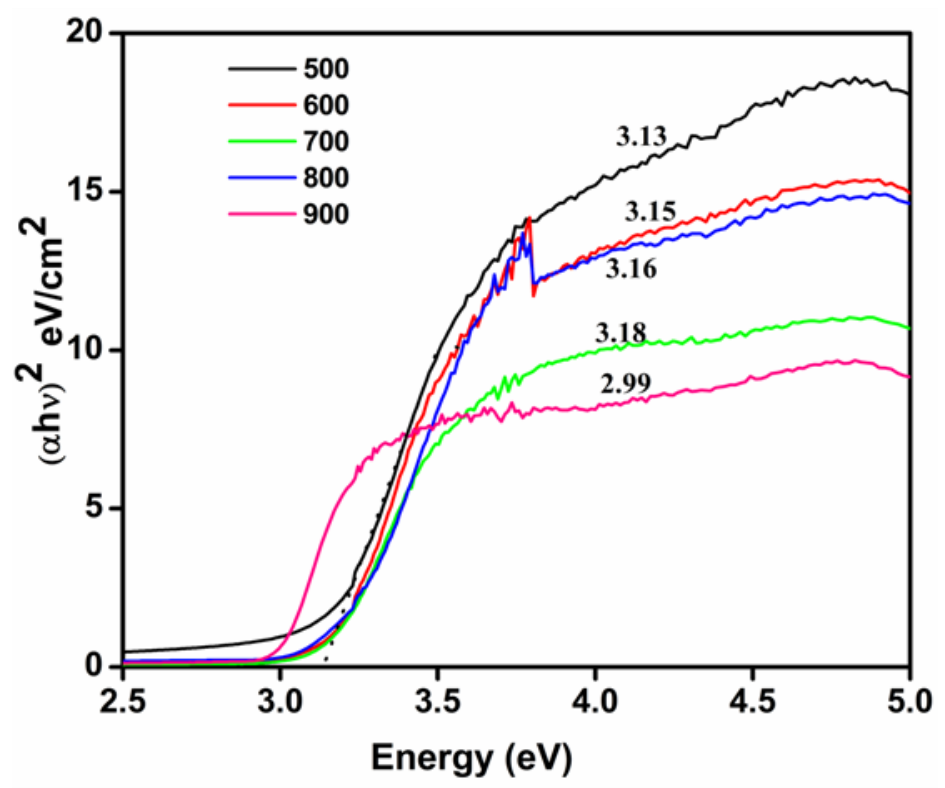

Figure 10. UV-Tauc plot of $\mathrm{TiO}_{2}$ nanoparticles calcined at different temperatures.

After the bulk, $\mathrm{TiO}_{2}$ had a band gap energy of $3.2 \mathrm{eV}$; the marginal reduction of $0.07 \mathrm{eV}$ in this parameter being attributed to particle size dependence [39]. The absorbance spectra and the corresponding Tauc plot of $\mathrm{TiO}_{2}$ nanoparticles calcined at different temperatures were as given in Figures 10 and 11, respectively.

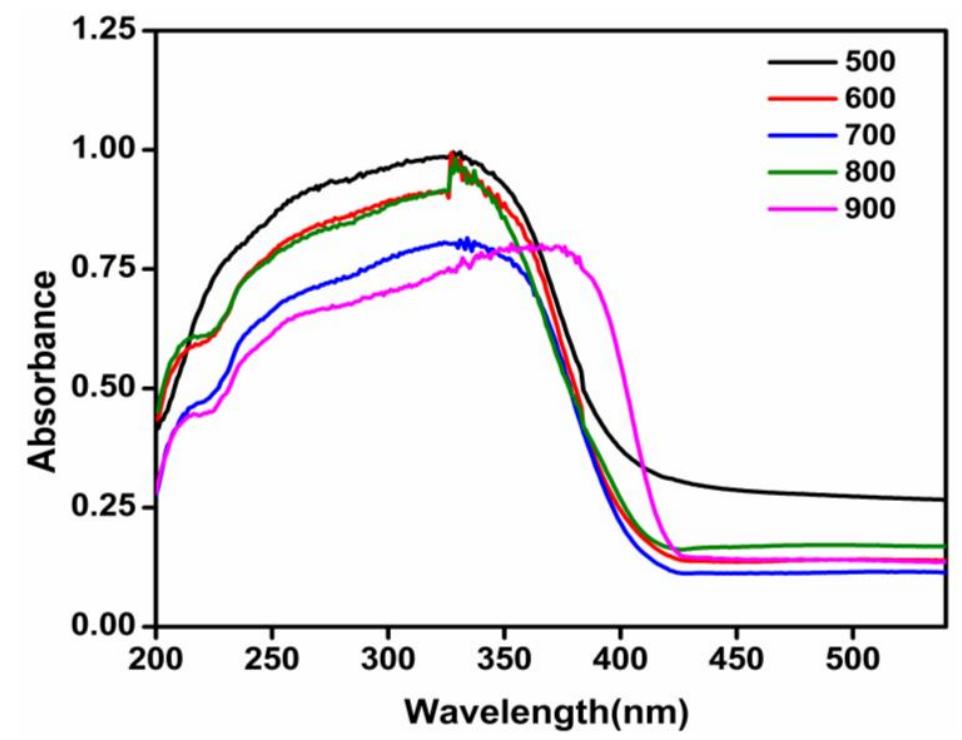

Figure 11. UV-Visible spectra of $\mathrm{TiO}_{2}$ nanoparticles calcined at different temperatures.

The calculated band gap of $\mathrm{TiO}_{2}$ nanoparticles, calcined at different temperatures was as shown in Table 1. It is also evident from the UV data (Table 1) that the band gap increased with the elevation of calcination temperatures from 500 to $800{ }^{\circ} \mathrm{C}$, before decreasing gradually at $900{ }^{\circ} \mathrm{C}$. This variation can be expected and is plausible, given that with an increase in the $\mathrm{TiO}_{2}$ calcination temperature, the anatase phase became endowed with good crystal characteristics. Furthermore, the decrease in band gap at $900{ }^{\circ} \mathrm{C}$ was mainly attributed to the complete formation of the rutile phase. 
Table 1. Band gap of $\mathrm{TiO} 2$ nanoparticles calcined at different temperatures.

\begin{tabular}{cc}
\hline Calcination Temperatures of $\mathrm{TiO}_{\mathbf{2}}\left({ }^{\circ} \mathbf{C}\right)$ & Band Gap (eV) \\
\hline 500 & 3.13 \\
600 & 3.15 \\
700 & 3.16 \\
800 & 3.18 \\
900 & 3 \\
\hline
\end{tabular}

\subsection{Photocatalytic Activity}

The photocatalytic activity of green-synthesized $\mathrm{TiO}_{2} \mathrm{NPs}$ was demonstrated by using an organic dye (methylene blue) under solar light, the dye degradation being initially identified by color change (Scheme 1). Additionally, we monitored the intensity of solar light throughout our experiment and confirmed it was in the range 100,000 Lux (see supplementary information, Figure S1). Furthermore, solar UV-radiation, as a function of time, and the UV intensity were measured using a Lux meter and UV filtering goggles. This information has been given in the supplementary document (Figures S2 and S3, respectively). The UV intensity from solar light was ascertained by calculating the difference between the intensity of solar radiation and the radiation through UV filtering goggles.

The dye displayed a distinct absorbance peak in visible light at a wavelength of $663 \mathrm{~nm}$, where absorbance was at a maximum. This peak was used to monitor dye concentration in the solution over time. From the absorbance spectra corresponding to dye degradation, it was indisputable that the presence of titanium dioxide nanocatalysts resulted in a linear increase of the percentage of dye degradation with time, reflected by the decrease in absorbance. This finding indicated that when the time of irradiation was prolonged, the percent degradation increased and reached a maximum after 90 min of solar irradiation. When $\mathrm{TiO}_{2}$ nanocatalysts, dispersed in the dye solution, were irradiated with solar radiation, photo-generated charge carriers induced redox reactions on the surface of the photocatalyst. Essentially, any contaminant adsorbed onto the photocatalyst surface, by virtue of electron-hole pair generation, will undergo either reduction or oxidation, respectively. Generally, the $\mathrm{TiO}_{2}$ photocatalyst surface behaves as an active center in photocatalysis, either through the generation of $\mathrm{OH}$ radicals (by oxidation of $\mathrm{OH}^{-}$anions) or by the generation of $\mathrm{O}_{2}{ }^{-}$radicals (via the reduction of $\mathrm{O}_{2}$ molecules) [40]. Subsequently, these photo-generated radicals and anions react with the adsorbed organic contaminants to degrade or mineralize them into less harmful by-products, such as $\mathrm{CO}_{2}$ and $\mathrm{H}_{2} \mathrm{O}$ (Scheme 1).

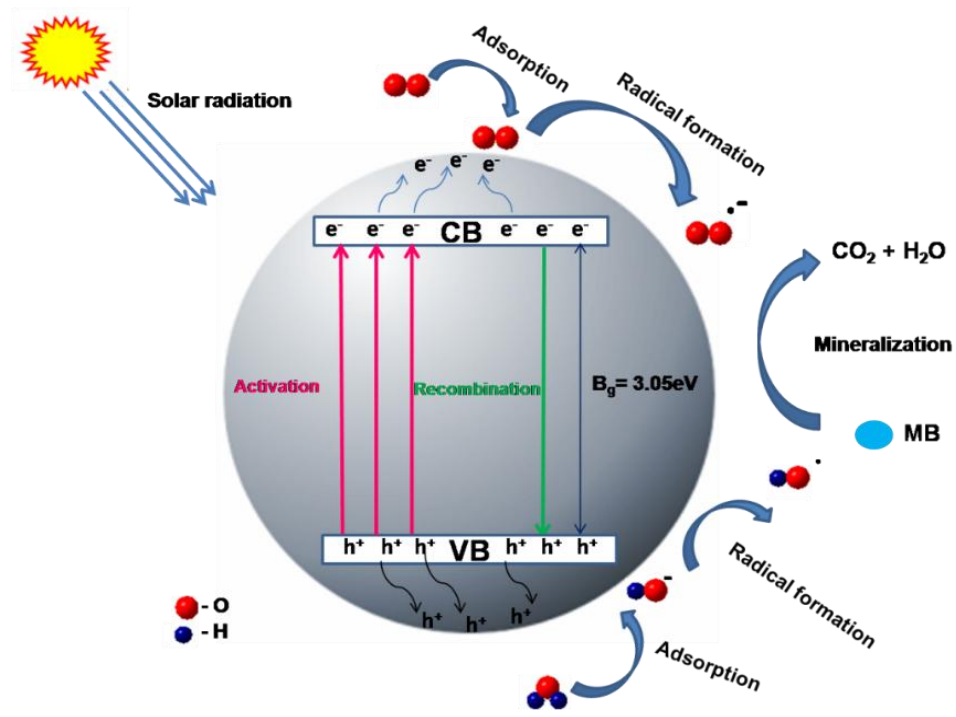

Scheme 1. Photocatalysis mechanism of $\mathrm{TiO}_{2}$. 
As the irradiation time lengthened, absorption of increasingly more light energy impinging on the catalyst surface occurred. This led to the increased formation of photo-excited species, and consequently, enhanced photocatalytic activity. From this study, it was observed that the photocatalytic dye degradation process was enhanced by lengthening exposure time (Figure 12). The Beer-Lambert-Bouguer law-relating the attenuation of light to the properties of the material through which the light is travelling-was used to determine molar concentrations of the degraded dyes.

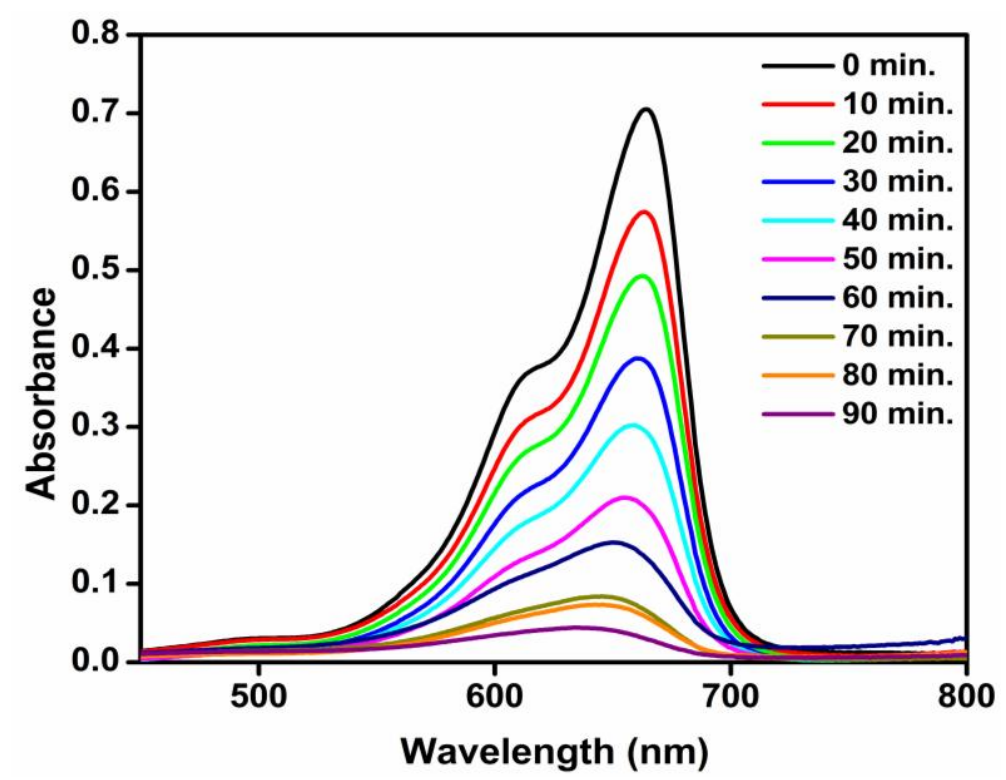

Figure 12. UV-visible spectra of methylene blue (MB) over various time intervals, in the presence of $\mathrm{TiO}_{2}$ nanocatalysts.

No degradation of dye was discerned in the absence of solar light (supplementary information; Figure S2). The small decrease in absorbance observed was due to the insignificant adsorption of dye molecules on the catalyst surface. We have conducted a control experiment with MB on its own, as shown in supplementary information Figure S4. From this data, it is clear that $\mathrm{TiO}_{2}$ was activated by solar light and was responsible for dye degradation. Moreover, photocatalytic degradation of dye was confirmed by the supplementary information (Figure S5, i.e., in the absence of sunlight, there is no degradation observed, confirming the major role of sunlight in the activation of the $\mathrm{TiO}_{2}$ nanocatalyst). From Figures S4 and S5, it was confirmed that dye discoloration was entirely due to photocatalytic degradation, not adsorption.

\subsubsection{Effect of Catalyst Concentration on Photocatalytic Activity of $\mathrm{TiO}_{2} \mathrm{Nanoparticles}$}

The effect of catalyst concentration on photocatalytic activity was tested by loading 1 to $15 \mathrm{mg} / 50 \mathrm{~mL}$ of $\mathrm{TiO}_{2}$ nanocatalyst in methylene blue solution. The photocatalytic degradation of methylene blue was highly influenced by the level of catalyst loading, as evident in Figure 13 which shows that the percent degradation of the dye increased with the amount (from 1 to $15 \mathrm{mg} / 50 \mathrm{~mL}$ ) of $\mathrm{TiO}_{2}$ nanocatalyst loading and remained virtually constant above a certain level. This is because as the amount of catalyst increased, a greater number of active sites on the photocatalyst surface became available. Consequently, more $\mathrm{OH}$ radicals were produced, which facilitated their participation in the dye degradation process. However, beyond a certain limiting value of catalyst loading, the solution appeared turbid. As a result, the passage of solar radiation into the reaction mixture (required for the reaction to proceed) was obstructed, and thus, the percent degradation of the dye decreased or remained constant [41]. 


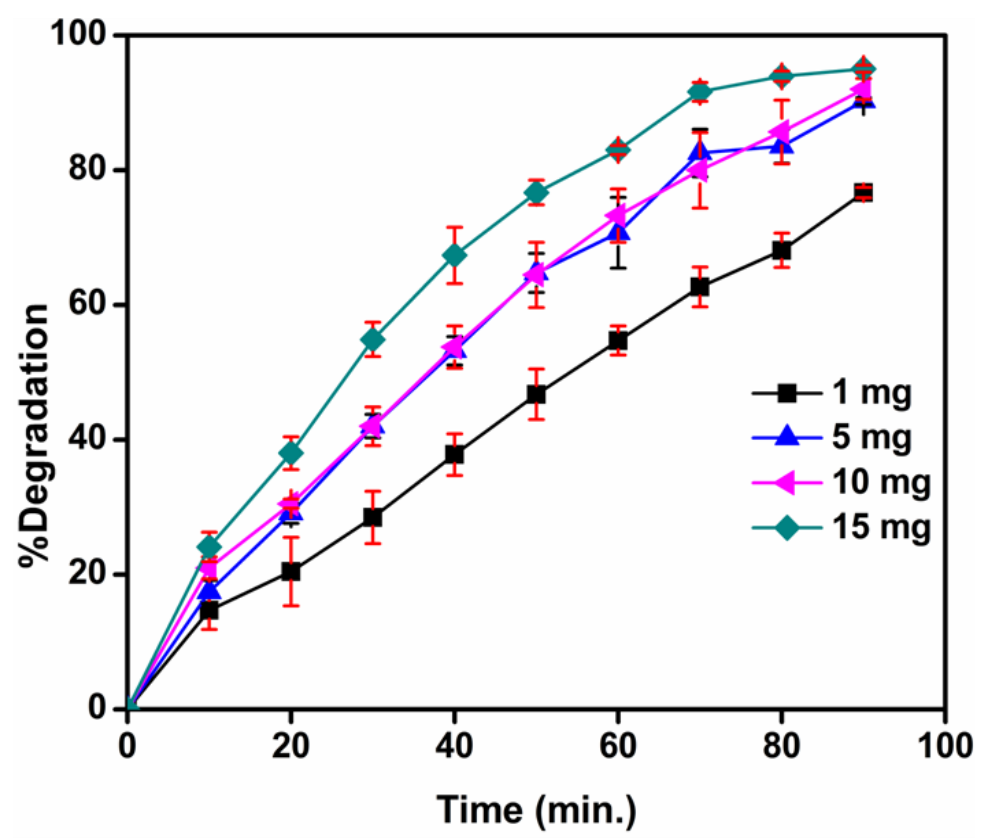

Figure 13. UV-visible spectra of $\mathrm{MB}$ dye $\left(10^{-5} \mathrm{M}\right)$ with different amounts of catalyst loading. Data obtained by degradation versus time are average values of three independent experiments (reported values $=$ mean \pm S.D.)

\subsubsection{Effect of $\mathrm{pH}$ on Photocatalytic Activity of $\mathrm{TiO}_{2}$ Nanoparticles}

The role of $\mathrm{pH}$ on the rate of photocatalytic degradation was studied over the $4-9 \mathrm{pH}$ range and the results are illustrated in Figure 14. It was observed that the percent degradation increased with a rising $\mathrm{pH}$, exhibiting a maximum between the $\mathrm{pH} 7-9$ ranges. This $\mathrm{pH}$ variation may bring about changes to the surface charge on the $\mathrm{TiO}_{2}$ nanoparticles and vary the potential associated with catalytic reactions. With the variation of potential, the extent of dye adsorption on the catalyst surface also fluctuates, culminating in the alteration of reaction velocity. Furthermore, under alkaline conditions, the surface of $\mathrm{TiO}_{2}$ could acquire a negative charge. Since methylene blue is a cationic dye and the surface of $\mathrm{TiO}_{2}$ nanoparticles in alkaline media attains a negative charge, the latter can be easily adsorbed onto the catalyst surface. This may lead to enhanced photocatalytic dye degradation under basic conditions [42]. The surface of $\mathrm{TiO}_{2}$ nanoparticles, in acidic or alkaline circumstances, can be protonated or deprotonated, respectively, according to the following reactions:

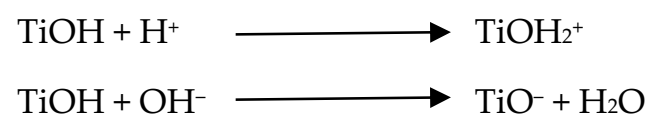




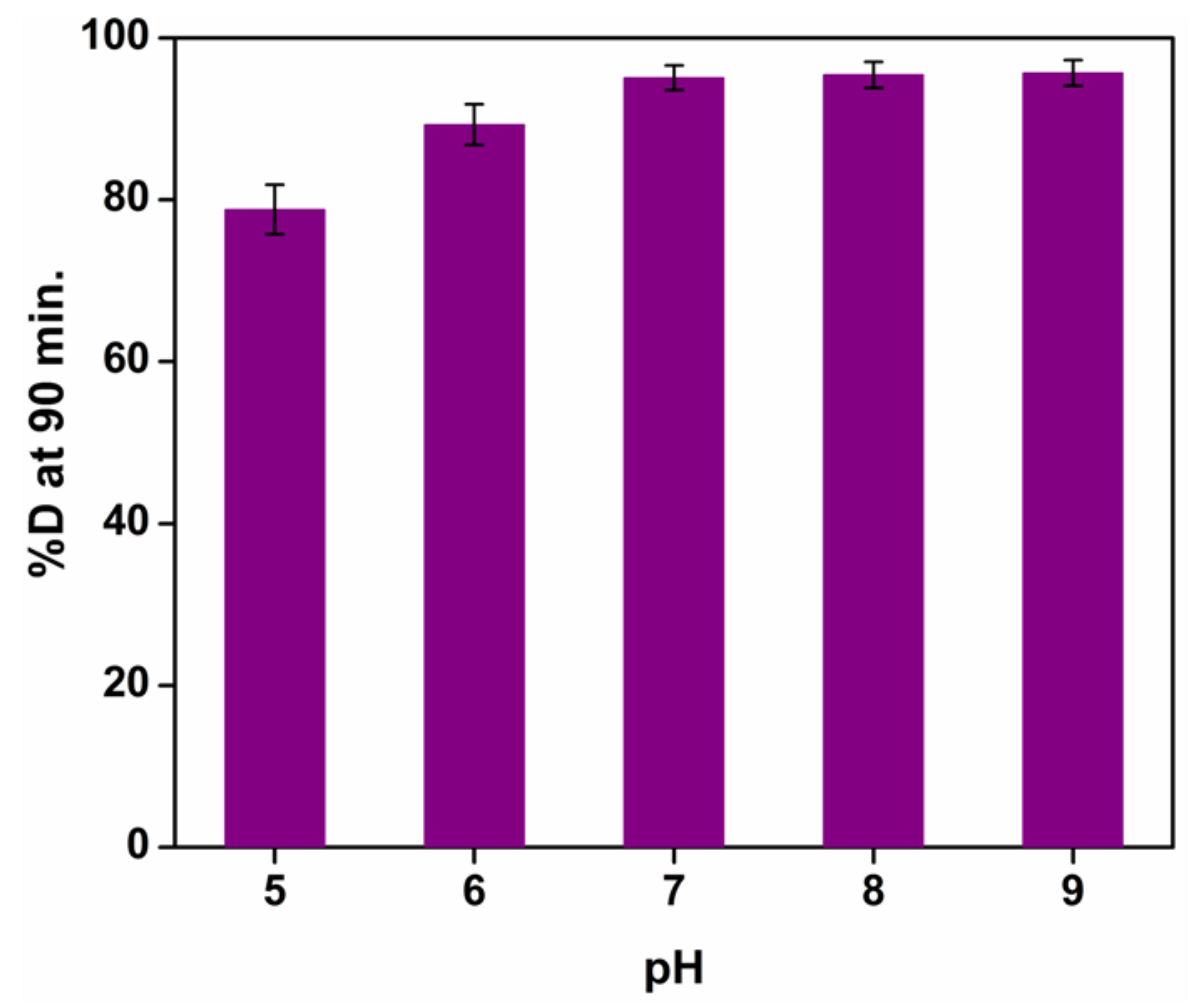

Figure 14. The photocatalytic degradation of $\mathrm{MB}$ dye $\left(10^{-5} \mathrm{M}\right)$ after treatment with photocatalyst $\mathrm{TiO}_{2}(0.2 \mathrm{~g} / \mathrm{L})$ in solar light under different $\mathrm{pH}$ conditions (reported values are for three independent experiments; average value $=$ mean \pm S.D; $n=3$ ).

\subsubsection{Effect of Calcination Temperature on Photocatalytic Activity of $\mathrm{TiO}_{2} \mathrm{Nanoparticles}$}

The effect of calcination temperatures of the $\mathrm{TiO}_{2}$ nanocatalyst on photocatalytic degradation is depicted in Figure 15. With increasing calcination temperatures of the $\mathrm{TiO}_{2}$ nanocatalyst, the percentage degradation was found to have risen and attained a maximum of $96.85 \%$ at $700{ }^{\circ} \mathrm{C}$. It then decreased gradually at temperatures between $800{ }^{\circ} \mathrm{C}$ and $900{ }^{\circ} \mathrm{C}$. This variation can be expected, given that an increase in calcination temperature of the $\mathrm{TiO}_{2}$ generated an anatase phase possessing good crystal characteristics-an essential criterion for photocatalytic degradation. It is also apparent from the UV data (Table 1) that the band gap increased with rising calcination temperatures, from $500{ }^{\circ} \mathrm{C}$ to $800^{\circ} \mathrm{C}$. Hence, the rate of electron-hole recombination decreased, and consequently, photocatalytic degradation increased. The decline in photocatalytic degradation at $800{ }^{\circ} \mathrm{C}$ was mainly ascribed to the formation of the rutile phase, which existed as a mixture of both the anatase and rutile forms. Complete formation of the rutile phase transpired at $900{ }^{\circ} \mathrm{C}$, and thus site activity fell and degradation decreased once again.

It is clear that the photocatalytic ability of titanium dioxide NPs is largely dependent on its crystalline form. Thermodynamically, the efficiency corresponding to photo-oxidation by the anatase and rutile phases should be similar. The surface recombination of photo-excited electrons and positive holes was higher in rutile than in anatase, which has a greater free (electron)-carrier mobility. Hence, a range of photoactivities was observed for each crystal form. This showed that variables such as crystal type and particulate sizes, as well as various synthesis routes (temperature, heating time etc.) largely determine its photocatalytic activity [40]. 


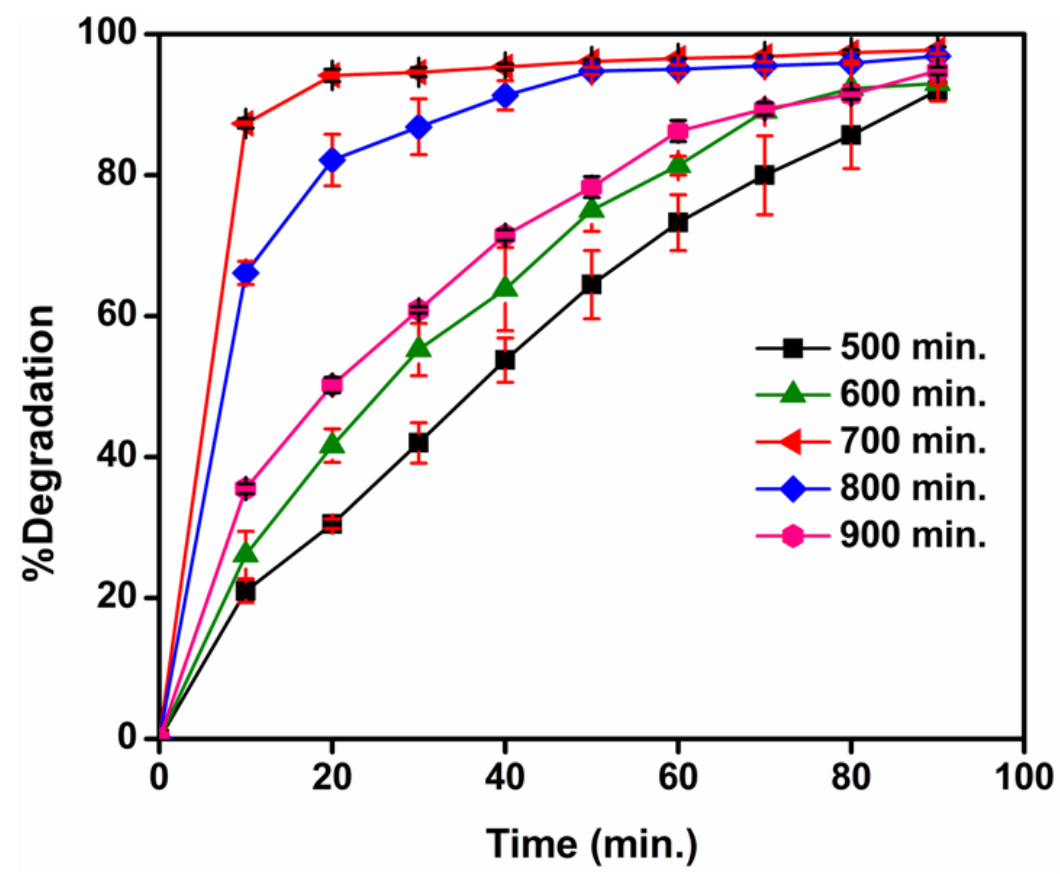

Figure 15. The photocatalytic degradation of $\mathrm{MB}$ dye $\left(10^{-5} \mathrm{M}\right)$ after treatment with photocatalyst $\mathrm{TiO}_{2}$ $(0.2 \mathrm{~g} / \mathrm{L})$ calcined at different temperatures under solar light (Values were reported as mean \pm S.D; $n=3)$.

\section{Conclusions}

Green nanotechnology has, in recent years, been accorded increasing importance for two reasons, namely, its contribution towards the elimination of harmful reagents and its ability to facilitate the synthesis of valuable products in a cost-effective manner. The green synthesis of $\mathrm{TiO}_{2} \mathrm{NPs}_{\text {involves }}$ more compatible, eco-friendly, low cost and less time-consuming processes compared to other synthetic methods such as the sol-gel technique, which has been widely used to achieve the same ends. In the present study, titanium dioxide nanoparticles were produced using a natural hydrocolloid, gum Kondagogu (Cochlospermum gossypium). The crystallinity and high purity of the synthesized $\mathrm{TiO}_{2}$ nanoparticles in the anatase form were unambiguously confirmed by the presence of sharp peaks (and the absence of unidentified peaks) in the X-Ray Diffraction patterns, Raman spectroscopic results and TEM images obtained. The absence of the original gum residue on the nanoparticles after calcination for $4 \mathrm{~h}$ at $500{ }^{\circ} \mathrm{C}$ was confirmed by FTIR, EDX, BET and TGA. The nanoparticles created had a mean particle size of approximately $11 \mathrm{~nm}$, a value which was determined by three independent techniques: Scherrer's formula from XRD (12.58 nm); TEM (8-13 nm) and CPS (11.2 $\pm 0.2 \mathrm{~nm})$, the figures in parentheses being the respective particle sizes. The photocatalytic activity of green-synthesized titanium dioxide nanoparticles was evaluated by adopting methylene blue dye as a model system. It is apparent that the photocatalytic effectiveness of titanium dioxide nanoparticles is largely dependent on various factors including its crystalline form, exposure time, extent of catalyst loading and solution $\mathrm{pH}$ conditions. The present study has demonstrated that titanium dioxide nanoparticles (synthesized through a green route by the use of natural, renewable and eco-friendly materials) exhibit excellent photocatalytic activity towards organic dye degradation. This system can be employed in water purification and dye effluent treatment.

Supplementary Materials: The following are available online at http:/ /www.mdpi.com/2079-4991/8/12/1002/ s1, Figure S1: The intensity of solar light measured throughout the experiment (range 100,000 Lux); Figure S2: The $\%$ of UV intensity in solar radiation at various intervals of time; Figure S3: The solar UV intensity measurement using Lux meter and UV filtering goggles; Figure S4: UV-visible spectra of MB over various time intervals after treatment with $\mathrm{TiO} 2$ nanocatalyst under dark condition; Figure S5: UV-visible spectra of MB over various time intervals in the absence of $\mathrm{TiO} 2$ nanocatalyst. 
Author Contributions: Formal analysis, S.W.; Funding acquisition, M.Č.; Investigation, K.S.; Methodology, C.S.; Project administration, M.Č.; Resources, R.P.; Supervision, B.G. and M.Č.; Writing-original draft, K.S.S.; Writing-review \& editing, V.V.T.P.

Funding: This research was funded including article processing charge (APC) by Ministry of Education, Youth and Sports of the Czech Republic and the European Union-European Structural and Investment Funds in the frames of Operational Program Research, Development and Education-project Hybrid Materials for Hierarchical Structures (HyHi, Reg. No. CZ.02.1.01/0.0/0.0/16_019/0000843).

Acknowledgments: The research reported in this paper was financially supported by the Ministry of Education, Youth and Sports in the framework OPR\&DI project 'Extension of CxI facilities' (CZ.1.05/2.1.00/19.0386). The authors' also acknowledge the assistance provided by the Research Infrastructure NanoEnviCz, under Project No. LM2015073 and Ministry of Education, Youth and Sports of the Czech Republic and the European Union-European Structural and Investment Funds in the frames of Operational Program Research, Development and Education-project Hybrid Materials for Hierarchical Structures (HyHi, Reg. No. CZ.02.1.01/0.0/0.0/16_019/0000843). The authors also gratefully acknowledge financial support from the Council for Scientific and Industrial Research, India.

Conflicts of Interest: The authors declare no conflict of interest.

\section{References}

1. Makarov, V.V.; Love, A.J.; Sinitsyna, O.V.; Makarova, S.S.; Yaminsky, I.V.; Taliansky, M.E.; Kalinina, N.O. “Green" Nanotechnologies: synthesis of metal nanoparticles using plants. Acta Naturae 2014, 6, 35-44. [PubMed]

2. Jana, A.; Scheer, E.; Polarz, S. Synthesis of graphene-transition metal oxide hybrid nanoparticles and their application in various fields. Beilstein J. Nanotechnol. 2017, 8, 688-714. [CrossRef] [PubMed]

3. Kim, C.S.; Moon, B.K.; Park, J.H.; Choi, B.C.; Seo, H.J. Solvothermal synthesis of nanocrystalline $\mathrm{TiO}_{2}$ in toluene with surfactant. J. Cryst. Growth 2003, 257, 309-315. [CrossRef]

4. Gotić, M.; Musić, S. Synthesis of nanocrystalline Iron oxide particles in the Iron(iii) acetate/alcohol/acetic acid system. Eur. J. Inorg. Chem. 2008, 6, 966-973. [CrossRef]

5. Hayashi, H.; Hakuta, Y. Hydrothermal synthesis of metal oxide nanoparticles in supercritical water. Materials 2010, 3, 3794-3817. [CrossRef] [PubMed]

6. Sharma, D.; Kanchi, S.; Bisetty, K. Biogenic synthesis of nanoparticles: A review. Arab. J. Chem. 2015. [CrossRef]

7. Lin, H.; Li, L.; Zhao, M.; Huang, X.; Chen, X.; Li, G.; Yu, R. Synthesis of high-quality brookite $\mathrm{TiO}_{2}$ single-crystalline nanosheets with specific facets exposed: tuning catalysts from inert to highly reactive. J. Am. Chem. Soc. 2012, 134, 8328-8331. [CrossRef] [PubMed]

8. Diebold, U. The surface science of titanium dioxide. Surf. Sci. Rep. 2003, 48, 53-229. [CrossRef]

9. Fernandez-Garcia, M.; Belver, C.; Hanson, J.C.; Wang, X.; Rodriguez, J.A. Anatase- $\mathrm{TiO}_{2}$ nanomaterials: analysis of key parameters controlling crystallization. J. Am. Chem. Soc. 2007, 129, 13604-13612. [CrossRef]

10. Fernandez-Garcia, M.; Rodriguez, J.A. Metal Oxide Nanoparticles; Brookhaven National Laboratory: Upton, NY, USA, 2007.

11. Bao, S.J.; Lei, C.; Xu, M.W.; Cai, C.J.; Cheng, C.J.; Li, C.M. Environmentally-friendly biomimicking synthesis of $\mathrm{TiO}_{2}$ nanomaterials using saccharides to tailor morphology, crystal phase and photocatalytic activity. CrystEngComm 2013, 15, 4694-4699. [CrossRef]

12. Vinod, V.T.P.; Sashidhar, R.B.; Černík, M. Morphology and metal binding characteristics of a natural polymer-kondagogu (Cochlospermum gossypium) gum. Molecules 2013, 18, 8264-8274. [CrossRef] [PubMed]

13. Vinod, V.T.P.; Wacławek, S.; Černík, M.; Varma, R.S. Tree gum-based renewable materials: Sustainable applications in nanotechnology, biomedical and environmental fields. Biotechnol. Adv. 2018, 36, 1984-2016.

14. Vinod, V.T.P.; Sashidhar, R.B. Surface morphology, chemical and structural assignment of gum Kondagogu (Cochlospermum gossypium DC.): An exudate tree gum of India. Indian J. Nat. Prod. Resour. 2010, 1, 181-192.

15. Vinod, V.T.P.; Sashidhar, R.B.; Sukumar, A.A. Competitive adsorption of toxic heavy metal contaminants by gum kondagogu (Cochlospermum gossypium): a natural hydrocolloid. Colloids Surf. B. 2010, 75, 490-495. [CrossRef] [PubMed]

16. Vinod, V.T.P.; Saravanan, P.; Sreedhar, B.; Devi, D.K.; Sashidhar, R.B. A facile synthesis and characterization of $\mathrm{Ag}$, $\mathrm{Au}$ and $\mathrm{Pt}$ nanoparticles using a natural hydrocolloid gum kondagogu (Cochlospermum gossypium). Colloids Surf. B. 2011, 83, 291-298. [CrossRef] [PubMed] 
17. Padil, V.V.T.; Cernik, M. Green synthesis of copper oxide nanoparticles using gum karaya as a biotemplate and their antibacterial application. Int. J. Nanomed. 2013, 8, 889-898. [CrossRef]

18. Vinod, V.T.P.; Sashidhar, R.B.; Suresh, K.I.; Rao, B.R.; Vijaya Saradhi, U.V.R.; Rao, T.P. Morphological, physico-chemical and structural characterization of gum kondagogu (Cochlospermum gossypium): A tree gum from India. Food Hydrocoll. 2008, 22, 899-915. [CrossRef]

19. Vinod, V.T.P.; Sashidhar, R.B.; Sarma, V.U.M.; Vijaya Saradhi, U.V.R. Compositional analysis and rheological properties of gum kondagogu (Cochlospermum gossypium): a tree gum from India. J. Agric. Food. Chem. 2008, 56, 2199-2207. [CrossRef]

20. Naidu, V.G.M.; Madhusudhana, K.; Sashidhar, R.B.; Ramakrishna, S.; Khar, R.K.; Ahmed, F.J.; Diwan, P.V. Polyelectrolyte complexes of gum kondagogu and chitosan, as diclofenac carriers. Carbohydr. Polym. 2009, 76, 464-471. [CrossRef]

21. Khade, G.V.; Suwarnkar, M.B.; Gavade, N.L.; Garadkar, K.M. Green synthesis of $\mathrm{TiO}_{2}$ and its photocatalytic activity. J. Mater. Sci. Mater. Electron. 2015, 26, 3309-3315. [CrossRef]

22. Kant, R. Textile dyeing industry an environmental hazard. Nat. Sci. 2012, 4, 22-26. [CrossRef]

23. Neyaz, N.; Siddiqui, W.A.; Nair, K.K. Application of surface functionalized Iron oxide nanomaterials as a nanosorbents in extraction of toxic heavy metals from ground water: a review. Int. J. Environ. Sci. 2014, 4, 472-483. [CrossRef]

24. Byrne, C.; Fagan, R.; Hinder, S.; McCormack, D.E.; Pillai, S.C. New approach of modifying the anatase to rutile transition temperature in $\mathrm{TiO}_{2}$ photocatalysts. RSC Adv. 2016, 6, 95232-95238. [CrossRef]

25. Carp, O.; Huisman, C.L.; Reller, A. Photoinduced reactivity of titanium dioxide. Prog. Solid State Chem. 2004, 32, 33-177. [CrossRef]

26. Mahlambi, M.M.; Ngila, C.J.; Mamba, B.B. Recent developments in environmental photocatalytic degradation of organic pollutants: the case of titanium dioxide nanoparticles-A review. J Nanomater. 2015, 2015, 1-29. [CrossRef]

27. Ahmed, M.A.; El-Katori, E.E.; Gharni, Z.H. Photocatalytic degradation of methylene blue dye using $\mathrm{Fe}_{2} \mathrm{O}_{3} / \mathrm{TiO}_{2}$ nanoparticles prepared by sol-gel method. J. Alloys Compd. 2013, 553, 19-29. [CrossRef]

28. Boury, B.; Plumejeau, S. Metal oxides and polysaccharides: An efficient hybrid association for materials chemistry. Green Chem. 2015, 17, 72-88. [CrossRef]

29. Dong, Y.; Wang, Y.; Cai, T.; Kou, L.; Yang, G.; Yan, Z. Preparation and nitrogen-doping of three-dimensionally ordered macroporous $\mathrm{TiO}_{2}$ with enhanced photocatalytic activity. Ceram. Int. 2014, 40, 11213-11219. [CrossRef]

30. Filippo, E.; Carlucci, C.; Capodilupo, A.L.; Perulli, P.; Conciauro, F.; Corrente, G.A.; Gigli, G.; Ciccarella, G. Enhanced photocatalytic activity of pure anatase $\mathrm{TiO}_{2}$ and $\mathrm{Pt}-\mathrm{TiO}_{2}$ nanoparticles synthesized by green microwave assisted route. Mater. Res. 2015, 18, 473-481. [CrossRef]

31. Haque, F.Z.; Nandanwar, R.; Singh, P. Evaluating photodegradation properties of anatase and rutile $\mathrm{TiO}_{2}$ nanoparticles for organic compounds. Optik 2017, 128, 191-200. [CrossRef]

32. Ba-Abbad, M.M.; Kadhum, A.A.H.; Mohamad, A.B.; Takriff, M.S.; Sopian, K. Synthesis and catalytic activity of $\mathrm{TiO}_{2}$ nanoparticles for photochemical oxidation of concentrated chlorophenols under direct solar radiation. Int. J. Electrochem. Sci. 2012, 7, 4871-4888.

33. Periyat, P.; Pillai, S.C.; McCormack, D.E.; Colreavy, J.; Hinder, S.J. Improved high-temperature stability and sun-light-driven photocatalytic activity of sulfur-doped anatase $\mathrm{TiO}_{2}$. J. Phys. Chem. C 2008, 112, 7644-7652. [CrossRef]

34. Reddy, G.B.; Madhusudhan, A.; Ramakrishna, D.; Ayodhya, D.; Venkatesham, M.; Veerabhadram, G. Green chemistry approach for the synthesis of gold nanoparticles with gum kondagogu: Characterization, catalytic and antibacterial activity. J. nanostructure chem. 2015, 5, 185-193. [CrossRef]

35. Chellappa, M.; Anjaneyulu, U.; Manivasagam, G.; Vijayalakshmi, U. Preparation and evaluation of the cytotoxic nature of $\mathrm{TiO}_{2}$ nanoparticles by direct contact method. Int. J. Nanomed. 2015, 10, 31-41. [CrossRef]

36. Ma, W.; Lu, Z.; Zhang, M. Investigation of structural transformations in nanophase titanium dioxide by Raman spectroscopy. Appl. Phys. A 1998, 66, 621-627. [CrossRef]

37. Ohsaka, T. Temperature dependence of the raman spectrum in anatase $\mathrm{TiO}_{2}$. J. Phys. Soc. Jpn. 1980, 48, 1661-1668. [CrossRef] 
38. Li, Y.; Qin, Z.; Guo, H.; Yang, H.; Zhang, G.; Ji, S.; Zeng, T. Low-temperature synthesis of anatase $\mathrm{TiO}_{2}$ nanoparticles with tunable surface charges for enhancing photocatalytic activity. PloS one 2014, 9, 1-19. [CrossRef]

39. Auvinen, S.; Alatalo, M.; Haario, H.; Jalava, J.P.; Lamminmäki, R.J. Size and shape dependence of the electronic and spectral properties in $\mathrm{TiO}_{2}$ nanoparticles. J. Phys. Chem. C 2011, 115, 8484-8493. [CrossRef]

40. Rauf, M.A.; Ashraf, S.S. Fundamental principles and application of heterogeneous photocatalytic degradation of dyes in solution. Chem. Eng. J. 2009, 151, 10-18. [CrossRef]

41. Wang, C.C.; Lee, C.K.; Lyu, M.D.; Juang, L.C. Photocatalytic degradation of C.I. Basic Violet 10 using $\mathrm{TiO}_{2}$ catalysts supported by Y zeolite: An investigation of the effects of operational parameters. Dyes. Pigm. 2008, 76, 817-824. [CrossRef]

42. Lakshmi, S.; Renganathan, R.; Fujita, S. Study on $\mathrm{TiO}_{2}$-mediated photocatalytic degradation of methylene blue. J. Photochem. Photobiol. A 1995, 88, 163-167. [CrossRef]

(C) 2018 by the authors. Licensee MDPI, Basel, Switzerland. This article is an open access article distributed under the terms and conditions of the Creative Commons Attribution (CC BY) license (http://creativecommons.org/licenses/by/4.0/). 